\title{
NOTE OF APPRECIATION / NOTE DE RECONNAISSANCE
}

The Editorial Board would like to thank the following reviewers for their contributions throughout 2004. Their expertise and dedication ensure that the Journal retains its high standards of quality. Their efforts are greatly appreciated by all those involved.
Le comité de rédaction tient à remercier les examinateurs mentionnés ci dessous de leur collaboration au cours de l'année 2004. Leur compétence et leur dévouement contribuent à maintenir les hautes normes de qualité de la revue. Leurs efforts sont grandement appréciés.

$\begin{array}{ll}\text { Alleman, J. } & \text { El-Sayrafi, O. } \\ \text { Alleman, T. } & \text { Erkey, J. } \\ \text { Anderson, W. } & \text { Ferguson, M. } \\ \text { Andrews, S. } & \text { Funamizu, N. } \\ \text { Awadallah, A. } & \text { Furumai, H. } \\ \text { Bagley, D. } & \text { Garvie, A. } \\ \text { Basu, O. } & \text { Gaskin, S. } \\ \text { Baudo, R. } & \text { George, D. } \\ \text { Beckie, R. } & \text { Gernaey, K. } \\ \text { Beltaos, S. } & \text { Glass, C. } \\ \text { Bielefeldt, A. } & \text { Granville, G. } \\ \text { Blackall, L. } & \text { Hall, K. } \\ \text { Boyd, G. } & \text { Inyang, H. } \\ \text { Bussière, B. } & \text { Johnson, A. } \\ \text { Cabral, A. } & \text { Johnson, S. } \\ \text { Campbell, H. } & \text { Jones, W. } \\ \text { Chauret, C. } & \text { Jordan, P. } \\ \text { Chieng, S. } & \text { Kennedy, C. } \\ \text { Chilibeck, B. } & \text { Kim, J. } \\ \text { Chu, A. } & \text { Kolka, R. } \\ \text { Cicek, N. } & \text { Koskinen, W. } \\ \text { Cunderlik, J. } & \text { Kruithof, J. } \\ \text { Davis, A. } & \text { Kuo, J. } \\ \text { Dixon, B. } & \text { Labat, J.A.B. } \\ \text { Dubé, J.-S. } & \text { LaPara, T. } \\ \end{array}$

Larney, F.

Layton, A.

Lee, $A$.

Lee, J.

Lefebvre, R.

Lemay, V.

Lunden, $M$.

McClellan, R.

McCray, J.

McKerchar, A.

Méhu, J.

Millette, L.

Mohseni, M.

Mohtar, R.H.

Moore, D.

Moore, R.D.

Moo-Young, $\mathrm{H}$.

Mulholland, P.

Mulligan, C.

Nema, A.K.

Niquette, P.

Ohlinger, $\mathrm{K}$.

Parker, W.

Peak, D.

Perrier, M.
Petrisor, I.

Qualls, R.

Ramkrishna, D.

Reckhow, D.

Safi, J.

Sanchez, F.

Sclimenti, M.

Sharp, T.

Shin, H.-S.

Singh, K.

Sommer, R.

Song, Y.C.

Spedding, G.

Spliid, N.H.

Stricker, A.-E.

Takizawa, S.

Tyagi, R.D.

Van Geel, P.

Verstraete, W.

Viraraghavan, $\mathrm{T}$.

Wang, S.C.

Wu, Q.

Xie, Y.

Yanful, E. 